\title{
Pontos Quânticos: Átomos Artificiais e Transistores Atômicos
}

\author{
Quantum Dots: Artificial atoms and atomic transistors \\ Adenilson J. Chiquito \\ Departamento de Física, Universidade Federal de São Carlos \\ Rodovia Washington Luiz, Km 235, CP 676, \\ 13565-905, São Carlos, São Paulo
}

Recebido em 12/03/2001. Aceito em 18/04/2001

\begin{abstract}
Os pontos quânticos podem ser estudados como átomos macroscópicos construídos artificialmente. Neste trabalho, são discutidos alguns conceitos básicos relacionados com o confinamento de elétrons em pontos quânticos, bem como alguns processos para sua obtenção. Uma argumentação simples é desenvolvida no sentido de mostrar algumas particularidades e aplicações dos pontos quânticos.

The quantum dots can be seen as giant man-made atoms. In this work, some basic concepts related to the electron confinement in quantum dots are discussed. The process of dots fabrication is also described. In addition, a simple discussion on the peculiar features and aplications of the quantum dots is presented.
\end{abstract}

\section{Introdução}

Desde 1947, após a demonstração do efeito transistor por J. Bardeen e W. Brattain em um cristal de germânio [1], os materiais semicondutores têm sido os responsáveis por inúmeros avanços, seja no desenvolvimento de novas tecnologias ou na área de pesquisa científica básica. Muitos destes avanços ocorreram a partir do aparecimento de estruturas híbridas por volta de 1970, as chamadas heteroestruturas [2]. Com o desenvolvimento e aprimoramento das técnicas de fabricação, como a epitaxia por feixe molecular ou MBE (molecular beam epitaxy), tornou-se possível o crescimento de camadas monoatômicas individuais uma após outra, produzindo redes cristalinas artificiais e interfaces quase perfeitas. Usando estas técnicas e a tecnologia de crescimento de cristais desenvolvida nas décadas de 80 e 90, muitas outras novas estruturas foram produzidas nas quais os efeitos de quantização são fundamentais. Um exemplo delas são os chamados pontos quânticos, nos quais os elétrons estão confinados nas três direções espaciais e devido a essa característica, muitas vezes nos referimos a eles como átomos gigantes [3]. A produção e o desenvolvimento dos pontos quânticos estão intimamente ligados à otimização de dispositivos eletrônicos já existentes, à fabricação de novos dispositivos eletrônicos e à miniaturização destes.

\section{Heteroestruturas e espectro de energia}

Uma heteroestrutura é basicamente a junção de materiais semicondutores diferentes. Quando dois semicondutores de gaps diferentes A e B são unidos atomicamente, como proporcionado pelo crescimento epitaxial, por exemplo, provoca-se uma descontinuidade nas bandas de energia da estrutura resultante [4], a qual comportase como um poço de potencial para o movimento de portadores na direção de crescimento da estrutura, confinando-os, como ilustrado na Fig. 1. Nesta, temos um esquema do diagrama de bandas de energia para os dois semicondutores (A e B) de gaps diferentes, isolados [Fig. 1(a)] e para uma junção em equilíbrio [Fig. 1(b)]. Na junção AB, partículas (elétrons) migram de um para outro material até que a condição de equilíbrio dada pela igualdade entre os potenciais químicos dos dois lados da junção, $\mu_{A}=\mu_{B}$ seja alcançada com o entortamento das bandas na região da interface. Assim, na interface temos

$$
E_{C A}=E_{C B}+\Delta E_{C}
$$

com $E_{\mathrm{CA}}$ e $\mathrm{E}_{\mathrm{CB}}$ representando o perfil da banda de condução para os materiais de cada lado da interface. 

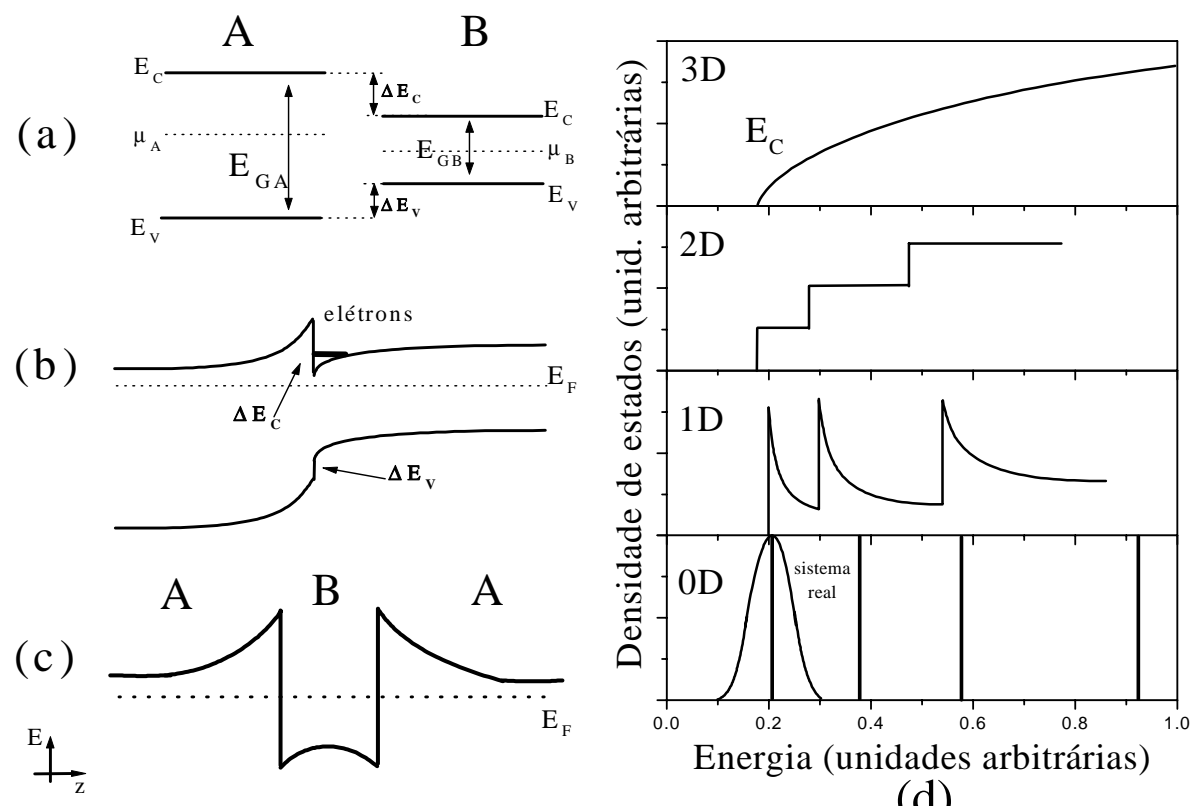

(d)

Figura 1. (a) Dois semicondutores separados, A e B, caracterizados pelo seus respectivos potenciais químicos $\left(\mu_{A}\right.$ e $\left.\mu_{B}\right)$, energia dos gaps $\left(\mathrm{E}_{\mathrm{GA}}\right.$ e $\left.\mathrm{E}_{\mathrm{GB}}\right)$ e descontinuidades das bandas $\left(\Delta \mathrm{E}_{\mathrm{C}}\right.$ e $\left.\Delta \mathrm{E}_{\mathrm{V}}\right)$; (b) quando unidos, ocorre troca de cargas $\mathrm{e}$ o sistema caminha para a situação onde há um potencial químico médio ou energia de Fermi ( $\left.\mathrm{E}_{\mathrm{F}}\right)$; (c) perfil da banda de condução em uma junção tipo ABA. Os potenciais químicos e a energia de Fermi podem ser entendidos como a energia limite até a qual os estados eletrônicos estão preenchidos (ver Ref. [18]). Em (d) mostra-se uma comparação entre as densidades de estados para sistemas zero-,uni,bi e tridimensionais.

Quando as dimensões de um sistema são comparáveis ao comprimento de onda de De Broglie $\left(\lambda_{B}\right)$, o movimento dos portadores torna-se quantizado, implicando em mudanças no espectro energético e nas propriedades dinâmicas dos portadores $[5,6]$. Se somente uma das direções é comparável a $\lambda_{B}$, diz-se que o sistema comporta-se dinamicamente como um sistema bidimensional (ou poços quânticos). Limitando o movimento em duas e depois em três direções, serão obtidos sistemas unidimensionais (ou fios quânticos) e zerodimensionais (ou pontos quânticos) $[7,8]$.

Para perceber esta situação é instrutivo comparar o comprimento de onda de De Broglie associado a um elétron e as dimensões características de um poço de potencial típico em uma heteroestrutura. Por exemplo, no sistema InAs/GaAs, o valor $\mathrm{E}=0,9 \mathrm{eV}$ é típico para o potencial de confinamento de elétrons (descontinuidade na banda de condução). O comprimento de De Broglie associado é dado por

$$
\lambda_{B}=\frac{\hbar}{\sqrt{2 m q V_{0}}}=\frac{1.23}{\sqrt{m^{*} V_{0}(\text { Volts })}}(\mathrm{nm})
$$

e usando $V_{0}=0,9 \mathrm{eV}$ e $m^{*}=0,023 m_{0}$ (massa efetiva para os elétrons no InAs) obtém-se $\lambda_{B} \approx 8 \mathrm{~nm}$, que é um valor bastante razoável se comparado às dimensões típicas de poços e pontos quânticos e neste caso, pelo menos um nível discreto poderá ser observado.
Imagine agora que construímos uma estrutura do tipo ABA. Neste caso, a forma da banda de condução da heteroestrutura ABA é tal como a representada na Fig. 1(c). É fácil reconhecer que temos um poço quântico finito como aqueles apresentados nos cursos de Mecânica Quântica (na junção tipo AB descrita acima, estávamos trabalhando com um poço aproximadamente triangular). Mais interessante ainda, é que as características deste poço podem ser controladas externamente: a profundidade do poço $\mathrm{V}_{0}$ (descontinuidade da banda de condução, $\Delta E_{C}$ ) depende apenas dos valores da energia dos gaps dos semicondutores escolhidos. Obviamente, a largura do poço também pode ser variada. Lembrando que as propriedades dos níveis eletrônicos permitidos num poço quântico dependem basicamente das características físicas do poço como largura e profundidade, temos então um versátil laboratório de Mecânica Quântica. Podemos descrever o comportamento de um elétron nesta estrutura usando a equação de Schrödinger

$$
\left[\frac{-\hbar^{2}}{2 m^{*}} \nabla^{2}+V(z)\right] \Psi(x, y, z)=E \Psi(x, y, z)
$$

com

$$
V(z)=\Delta E_{C}+\Phi(x, y, z) .
$$


$E$ é a energia total do nível quantizado, $\Psi(x, y, z)$ é a função de onda associada a $E$ e $\Phi$ é o potencial devido à redistribuição de cargas na interface como representado pela curvatura das bandas próximo às interfaces na Fig. 1(c) [4].

A presença de um poço quântico altera o espectro energético acessível aos elétrons dentro do poço e agora somente alguns valores discretos de energia são permitidos. Isso significa que para cada estado permitido, os elétrons podem mover-se livremente na direção $x-y$, mas não na direção $z$. A energia correspondente a um dado estado nesta estrutura é dada por

$$
E=E_{n z}+\frac{\hbar^{2}}{2 m^{*}}\left(k_{x}^{2}+k_{y}^{2}\right)
$$

onde o primeiro termo é relativo à energia dos níveis discretos na direção $z$. O outro termo descreve a energia nas direções $x$ e $y$. Note que o movimento dos elétrons e a energia associada tornaram-se quantizadas e o sistema comporta-se dinamicamente como um sistema bidimensional.

Com base nas considerações anteriores, para obter um potencial que confine os elétrons nas três direções espaciais ou um ponto quântico basta desenvolver um método que limite o movimento dos elétrons também nas direções $x$ e $y$ da estrutura ABA. Este é o assunto da próxima seção.

\section{Pontos Quânticos - como construí-los?}

Como mencionado anteriormente, a presença de um ponto quântico define um potencial tridimensional e o movimento eletrônico fica limitado nas três direções espaciais, $x, y$ e $z$. A palavra "ponto" sugere uma quantidade muito pequena a ser ocupada no espaço; apesar disso, um ponto quântico possui milhões de átomos reais e elétrons associados. A maior parte dos elétrons está fortemente ligada aos átomos que formam a estrutura do ponto quântico e somente algumas centenas deles estão livres.

Um dos métodos de fabricação usa uma estrutura composta por três materiais crescidos seqüencialmente (ABA), na qual o movimento eletrônico é confinado na direção de crescimento da estrutura (direção $z$ ) como mostrado na seção anterior. Para limitar o movimento dos elétrons no plano $x-y$ são construídas as chamadas "mesas" ou ilhas por meio de técnicas de litografia. Neste processo, uma máscara padrão é depositada sobre o semicondutor e em seguida, o sistema máscara + semicondutor é submetido a uma solução química que define a estrutura de mesas, formando caixas ou cilindros. Quando as dimensões desejadas para um dispositivo são pequenas (da ordem de $100 \mathrm{~nm}$ ou menos), usa-se litografia por feixes eletrônicos [9]. Tipicamente as mesas têm uma dimensão lateral $\left(L_{x, y, z}\right.$ da ordem de 100 nm - 50 nm [Fig. 2(a)].

Existe uma outra técnica de formação das ilhas através de um processo no qual os pontos quânticos constroem-se por si mesmos. Pontos quânticos autoorganizados de InAs inseridos em uma matriz de GaAs são um dos sistemas mais estudados atualmente, mas outros tipos também podem ser fabricados, como por exemplo, pontos de Ge inseridos em uma matriz de Si.

Existem três modos de crescimento epitaxial possíveis quando os materiais usados têm parâmetros de rede (espaçamento entre átomos na rede cristalina) diferentes: o modo Frank-van der Merve, o modo StranskiKrastanow e o modo Volmer-Weber [10]. No primeiro, o crescimento de um material sobre outro (como numa estrutura ABA) é feito camada por camada, enquanto que no último, o sistema somente torna-se estável se houver a formação de ilhas, ou nucleação. O segundo caso é um processo intermediário no qual há a formação de algumas camadas completas (cobrindo toda a superfície do substrato) até uma espessura crítica antes da formação dos pontos quânticos. A auto-organização dos átomos de In e As criando ilhas tridimensionais de InAs se processa no modo Stranski-Krastanow [11]. Os detalhes do processo de crescimento são bastante complexos e não totalmente compreendidos ainda, mas podemos descrevê-lo simplificadamente da seguinte forma: os materiais InAs e GaAs têm parâmetros de rede que diferem por aproximadamente 7\%. Quando se "cresce" InAs sobre GaAs aparece uma tensão na interface entre os dois materiais [12]. Isto origina uma força (driving force) que age no sentido de "casar" localmente as redes do GaAs e do InAs, dando origem a ilhas tridimensionais de InAs como as mostradas na Fig. 2(b). Nesta amostra temos aproximadamente $10^{10}$ pontos quânticos por centímetro cúbico, com uma base variando entre 10 e $25 \mathrm{~nm}$ de diâmetro [13]. Para a construção de um dispositivo qualquer, completa-se a estrutura da Fig. 2(b), com o crescimento de outra camada de GaAs sobre as ilhas de InAs, resultando em uma estrutura tipo ABA com $\mathrm{A}=$ GaAs e $\mathrm{B}=$ InAs (agora, B representa ilhas tridimensionais e não camadas completas). Do ponto de vista da energia total do sistema, a formação de ilhas pode ser associada à busca pela situação de equilíbrio com minimização da energia total.

Em resumo, os pontos quânticos auto-organizados são o resultado de forças originadas pela tentativa de crescimento de materiais com parâmetros de rede diferentes.

Até aqui falamos apenas de pontos quânticos construídos com base em semicondutores, mas também é necessário lembrar dos chamados pontos quânticos metálicos, onde pequenas ilhas de alumínio ou ouro são introduzidos em filmes isolantes como $\mathrm{Al}_{2} \mathrm{O}_{3}$ ou $\mathrm{Si}_{3} \mathrm{~N}_{4}$ [14]. 


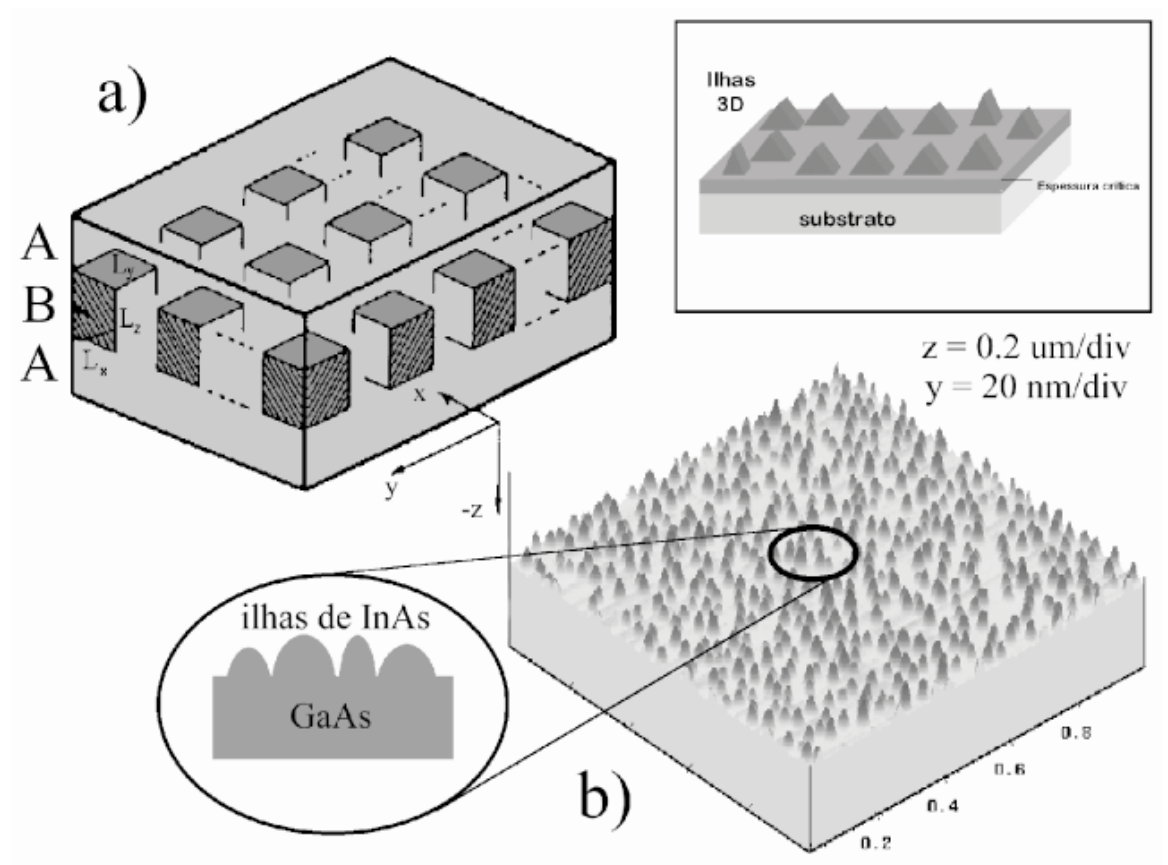

Figura 2. Na parte (a) um esquema de pontos quânticos construídos através de litografia por feixes eletrônicos [3] (note a estrutura tipo ABA). Uma fotografia de um sistema de pontos quânticos auto-organizados de InAs crescidos sobre GaAs pode ser vista em (b). Esta fotografia foi realizada por um microscópio de força atômica na Universidade da Pennsylvania, por um de nossos colaboradores, N. T. Moshegov [13]. Na inserção está uma representação do processo de formação dos pontos quânticos, quando o modo de crescimento da camada epitaxial passa de bidimensional a tridimensional, com o aparecimento dos pontos quânticos auto-organizados.

\section{III.1 Espectro de energia - pontos quânticos litográficos e auto-organizados}

O potencial mais adequado para descrever os pontos quânticos fabricados por litografia pode ser cilíndrico ou cúbico. Por outro lado, o potencial dos pontos quânticos auto-organizados pode ser tratado como hemisférico ou piramidal [Fig. 2(b)].

Modelando os pontos quânticos como caixas tridimensionais, por exemplo, podemos determinar os estados eletrônicos através da equação de Schrödinger [Eq.
(3)], na qual o potencial é definido por uma caixa de lado $L$. Como estamos interessados em características qualitativas, vamos considerar que o potencial é infinito fora da caixa e zero no seu interior. O primeiro passo para resolver a Eq. (3) com este potencial é tentar uma separação de variáveis, escrevendo a função de onda como

$$
\Psi(x, y, z)=X(x) \cdot Y(y) \cdot Z(z) .
$$

Substituindo a eq. (6) na equação de Schrödinger [Eq. (3)] e dividindo o resultado por $\Psi(x, y, z)$, obtemos

$$
\left[\frac{1}{X} \frac{d^{2} X}{d x^{2}}-\frac{2 m}{\hbar^{2}} V_{x}(x)\right]+\left[\frac{1}{Y} \frac{d^{2} Y}{d y^{2}}-\frac{2 m}{\hbar^{2}} V_{y}(y)\right]+\left[\frac{1}{Z} \frac{d^{2} Z}{d z^{2}}-\frac{2 m}{\hbar^{2}} V_{z}(z)\right]+\frac{2 m}{\hbar^{2}} E=0
$$

$\operatorname{com} V(x, y, z)=V_{x}(x)+V_{y}(y)+V_{z}(z)$. É conveniente, também, expressar a energia na forma $E=E_{x}+E_{y}+E_{z}$ [15].

Como a função $X(x)$ depende exclusivamente de $x$, uma mudança em $y$ ou $z$ não irá alterar o primeiro termo da Eq. (7). Similarmente, esta consideração vale para $y$ e $z$. Temos então, três equações diferenciais uni- dimensionais do tipo

$$
\frac{d^{2} X}{d x^{2}}=-\frac{2 m}{\hbar^{2}}\left[E_{x}-V_{x}(x)\right] X
$$

A solução de cada uma das equações é bastante simples (ver, por exemplo, Ref. [15]). Para a variável $x$, obtém-se: 


$$
X(x)=\sqrt{\frac{2}{L}} \operatorname{sen}\left(\frac{n_{x} \pi}{L} x\right) \operatorname{com} E_{x}=\frac{\hbar^{2}}{2 m} \frac{\pi^{2}}{L^{2}} n_{x}^{2} .
$$

Resultados similares podem ser encontrados para $y$ e $z$. Assim, a solução final fica [Eq. (6)]:

$$
\Psi(x, y, z)=\sqrt{\frac{8}{L^{3}}} \operatorname{sen}\left(\frac{n_{x} \pi}{L} x\right) \cdot \operatorname{sen}\left(\frac{n_{y} \pi}{L} y\right) \cdot \operatorname{sen}\left(\frac{n_{z} \pi}{L} z\right)
$$

e

$$
E_{n_{x} n_{y} n_{z}}=E_{x}+E_{y}+E_{z}=\frac{\hbar^{2}}{2 m} \frac{\pi^{2}}{L^{2}}\left[n_{x}^{2}+n_{y}^{2}+n_{z}^{2}\right]
$$

$\operatorname{com} n_{x}=0,1,2 \ldots, n_{y}=0,1,2 \ldots$ e $n_{z}=0,1,2 \ldots$ Compare agora as Eqs. (5) e (11): a primeira mostra que temos quantização parcial da energia (somente na direção de crescimento da estrutura). Na outra equação, a energia foi completamente discretizada nas três direções e é também por essa característica que se pode dizer que um ponto quântico é um sistema análogo aos átomos naturais.

Para tratar o caso dos sistemas auto-organizados, temos que mudar a forma do potencial para depois escrever a equação de Schrödinger. Observando a Fig. 1(b), vê-se que o sistema auto-organizado é constituído de ilhas hemisféricas e o potencial pode ser escrito como (lembre-se de um oscilador harmônico bidimensional)

$$
V_{x y}=\underbrace{\frac{1}{2} k\left[x^{2}+y^{2}\right]}_{\text {plano } x-y}+\underbrace{\mathrm{V}(\mathrm{z})}_{\text {direção } z},
$$

onde $k=\omega^{2} m$ é a constante restauradora, suposta isotrópica nas direções $x, y$. Usando o procedimento de separação de variáveis, obtemos equações diferenciais que descrevem um oscilador harmônico unidimensional nas direções $x$ e $y$. As soluções são proporcionais aos polinômios de Hermite [16]. A função de onda [Eq. (6)] para o estado fundamental do ponto quântico fica

$$
\Psi(x, y, z)=\frac{Z(z)}{\sqrt{\pi} R_{0}} \cdot \exp \left[\frac{-\left(x^{2}+y^{2}\right)}{2 R_{0}^{2}}\right]
$$

$\operatorname{com} R_{0}=\sqrt{\frac{\hbar}{m \omega_{0}}}$. A parte em $z$ é similar àquela descrita no caso anterior e, portanto, a energia total de um estado eletrônico num ponto quântico auto-organizado fica descrita por

$$
E_{n_{x} n_{y} n_{z}}=\left(n_{x}+n_{y}+1\right) \hbar \omega_{0}+E_{n_{z}} .
$$

Novamente, obtém-se quantização da energia nas três direções. Observe que $E_{n_{z}}$ refere-se à energia de quantização na direção $z$, como na Eq. (5). Tanto no caso do potencial caixa como do hemisférico, uma característica importante pode ser observada: como em átomos, há uma separação muito bem definida entre os níveis de energia, mas agora, todo o sistema é "manipulável". Em um átomo natural, o espectro de energias quase não pode ser alterado, principalmente porque é determinado pela interação entre os elétrons e a rede periódica de átomos e entre elétrons. Um ponto quântico, por sua vez, pode ter seu espectro de energia característico completamente alterado apenas se mudarmos a geometria e a composição dos materiais que o constituem (veja as equações acima). Note que é formada uma estrutura de subníveis como nos átomos naturais: é freqüente chamar o estado fundamental dos pontos quânticos como estado tipo $s\left(n_{x} n_{y} n_{z}=100\right) \mathrm{e}$ os outros estados como tipo $p, d, f$...etc.

O tamanho característico de um ponto quântico pode variar muito (por exemplo, desde $5 \mathrm{~nm}$ até 100 $\mathrm{nm}$ ) permitindo que o número de elétrons contidos nele varie desde zero até dezenas ou centenas. Esta característica permite o estudo do comportamento dos pontos quânticos em função do número de elétrons buscando, por exemplo, determinar se um sistema de um único elétron é fundamentalmente diferente de um sistema com muitos elétrons. Ainda com relação ao tamanho dos pontos quânticos, é possível a exploração de regimes não acessíveis em sistemas atômicos. Como exemplo, é possível conseguir um campo magnético através de um ponto quântico com um fluxo de $1 \mathrm{~T}$ por micrometro quadrado em um laboratório. Agora, para conseguir o mesmo fluxo através de um átomo, seria necessário um campo da ordem de 100.000.000 T!

Na próxima seção serão discutidas algumas propriedades e aplicações dos pontos quânticos.

\section{Algumas propriedades e aplicações dos pontos quânticos}

É possível obter muitas informações sobre átomos naturais e suas propriedades medindo as energias necessárias para ceder ou remover elétrons, o que é usualmente feito por técnicas de espectroscopia [17]. Em átomos artificiais também é muito importante o conhecimento destas energias, mas neste caso, as informações podem ser conseguidas através da medida da corrente através dos pontos quânticos. Vamos descrever algumas propriedades dos pontos quânticos, relacionadas com a ocupação dos estados eletrônicos e com a condução de corrente elétrica. 
Uma propriedade sempre interessante a ser considerada é a densidade de estados eletrônicos. Esta função descreve o número de estados possíveis na banda de condução (ou valência) por unidades de energia e volume que podem ser preenchidos com elétrons. Não será feita aqui uma derivação rigorosa da densidade de estados, como a encontrada na Ref. [18], mas podemos expressá-la na forma:

$N(E) d E=2 \times\left(\frac{\text { número de estados entre } E \text { e } E+d E}{V}\right)$,

onde $V$ representa o volume total do sistema. Usando uma dispersão parabólica de energia $\left(E=\frac{\hbar^{2} k^{2}}{2 m}\right)$, as densidades de estados para os casos tridimensional (material homogêneo), bidimensional (por exemplo, estrutura ABA), unidimensional (fio quântico) e zerodimensional (ponto quântico) podem ser escritas como:

$$
\begin{aligned}
& N_{3 D}(E)=\frac{1}{2 \pi^{2}}\left[\frac{2 m}{\hbar^{2}}\right]^{3 / 2} \sqrt{E} \\
& N_{2 D}(E)=\sum_{E \geq E_{\subset}} \frac{m}{\pi \hbar^{2}} ; \\
& N_{1 D}(E)=\sum_{E \geq E_{\subset}} \frac{1}{\pi \hbar} \sqrt{\frac{m}{2\left(E-E_{\subset}\right)}} ; \\
& N_{0 D}(E)=2 \cdot \sum_{\subset} \delta\left(E-E_{\subset}\right),
\end{aligned}
$$

onde $E_{\subset}$ representa um nível permitido. Note principalmente a dependência em energia para cada um dos casos acima. Na Fig. 1(d) temos uma comparação entre as expressões acima. No caso 0D, em conseqüência de não haver movimento eletrônico, também não há um volume a ser ocupado no espaço recíproco [18]. Cada estado quântico desse sistema pode ser ocupado somente por dois elétrons (compare com átomos) e a densidade de estados é descrita por uma função delta de Dirac [Eq. (19)]. No caso de um sistema real, no qual nem todos os pontos quânticos são idênticos, temos uma distribuição aleatória de energias possíveis. Então é razoável substituir a função delta de Dirac por uma distribuição, por exemplo, gaussiana [Fig. 1(d)].

A utilização de sistemas de baixa dimensionalidade na fabricação de dispositivos eletrônicos e optoeletrônicos está diretamente ligada às características peculiares apresentadas por exemplo, pela densidade de estados. Quanto mais estreita for a distribuição de estados eletrônicos em energia, menor será a influência dos efeitos da temperatura e assim, a separação muito bem definida entre os níveis de energia em um ponto quântico, permite uma grande seletividade em termos de energia. Os pontos quânticos, portanto, são estruturas muito favoráveis ao desenvolvimento de novos dispositivos.

Como estamos falando da ocupação dos estados confinados com elétrons, também é interessante discutir os processos de carga e descarga dos pontos quânticos. Estes processos dependem basicamente de duas energias características: aquela relacionada com a interação coulombiana e outra ligada ao confinamento espacial dos elétrons. O efeito energético líquido de um elétron entrando ou saindo de um ponto quântico, como na estrutura InAs/GaAs pode ser estimado calculando-se a capacitância (ou auto-capacitância) do sistema. Considerando que um ponto quântico possa ser representado por uma esfera de raio $\mathrm{R}$ e lembrando que $\mathrm{Q}=\mathrm{CV}$, a auto-capacitância desse sistema é dada por

$$
C_{d o t}=4 \pi \epsilon \epsilon_{0} R \simeq 7.2 \times 10^{-18} \mathrm{~F},
$$

para ponto quântico com $R=5 \mathrm{~nm}\left(\epsilon, \epsilon_{0}\right.$ são as permissividades relativa do InAs e absoluta do vácuo, respectivamente [19]). Então, a energia eletrostática envolvida é

$$
E_{\text {Coul }}=\frac{e^{2}}{2 C_{d o t}}=\frac{e^{2}}{8 \pi \epsilon \epsilon_{0} R} \simeq 11 \mathrm{meV}
$$

(compare com a energia térmica à temperatura ambiente: $k_{B} T=25 \mathrm{meV}$ ). Esta energia tem um papel bastante importante no estudo das propriedades e aplicações dos pontos quânticos como será explorado na seção seguinte.

\section{IV.1 O transistor quântico (Single Electron Transistor, SET)}

Já em 1911, com o seu famoso experimento da gota de óleo, Millikan observou os efeitos de cargas individuais [20]. Em sólidos, a primeira observação dos efeitos de tunelamento de cargas individuais foi realizada em 1951 por Gorter [21]. Num recente trabalho, publicado nesta revista [1], foi dada uma breve descrição histórica do desenvolvimento do transistor e também foi discutido o princípio de funcionamento deste dispositivo. Agora, vamos estudar uma versão muito mais avançada de transitores baseada nas propriedades apresentadas por sistemas eletrônicos, quando elétrons são controlados individualmente.

Agora imagine um dispositivo como o representado na Fig. 3(a) e suponha que o nível discreto no ponto quântico já esteja ocupado com um elétron. Na Fig. 3(b) vê-se o diagrama de energia versus distância mostrando o potencial ao qual um elétron, no dispositivo representado na Fig. 3(a), está sujeito. Note que a região do ponto quântico, representada apenas por um 
poço quântico finito na Fig. 3, na verdade apresenta confinamento tridimensional para os elétrons.

Para haver corrente na estrutura (Fig. 3) é necessário que elétrons do contato 1 (ou 2) ultrapassem o potencial das barreiras, ou seja, a passagem dos elétrons do contato 1 através da barreira V1 para o nível de energia quantizado (ponto quântico); em seguida, a passagem do nível quantizado para o contato 2 através da barreira V2. A presença de um estado totalmente quantizado entre os contatos 1 e 2 , definido pelo ponto quântico, torna o transporte de cargas na estrutura bastante interessante porque somente um elétron (dois, considerando spin) pode ocupar o estado discreto dentro do ponto quântico, como é descrito pela energia nas Eqs. (11) e (14) e pela densidade de estados na Eq. $(19)^{1}$. Quando se tenta carregar os pontos quânticos com elétrons ${ }^{2}$ a interação elétron-elétron, quebra a degenerescência original dos níveis de energia dentro do ponto quântico e aparece um intervalo de energia (gap) e nesta nova situação, nenhum elétron pode tunelar para ou do ponto quântico como mostrado no lado esquerdo da Fig. 3(b).

(a)

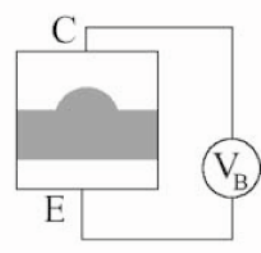

(b)

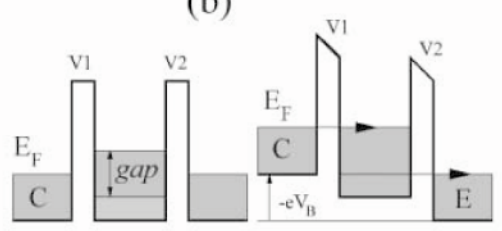

Figura 3. (a) Dispositivo com um ponto quântico inserido em material isolante ou semicondutor e polarizado com $V_{B}$; Em (b), está o diagrama do potencial ao qual estão sujeitos os elétrons do sistema com e sem aplicação de $V_{B}$. Note que o nível de energia dentro do ponto quântico foi separado em outros dois por uma energia $\Delta E$. Em (c) está a curva de corrente versus $\mathrm{V}_{\mathrm{B}}$ resultante.

Para transferir um elétron para o ponto quântico é necessária a energia descrita pela Eq. (21). Desprezando os efeitos térmicos, a única fonte de energia é a bateria $V_{B}$. Se a voltagem da bateria for menor que

$$
\Delta V_{\text {Coul }}=\frac{E_{\text {Coul }}}{e}
$$

(onde -e é a carga eletrônica), nenhum elétron será transferido $(\mathrm{C} \rightarrow \mathrm{E})$ porque não existem elétrons no sistema com energia suficiente para vencer a barreira coulombiana imposta por $\Delta V_{\text {Coul }}$. Aumentando a voltagem da bateria $V_{\mathrm{B}}$, será possível promover o tunelamento como indicado à direita na Fig. 3(b) pelas setas, dando origem a uma corrente entre C e E. Na Fig. 3(c) observamos a curva característica da corrente correspondente à situação descrita acima: quando o efeito coulombiano age, a corrente é nula enquanto que para $V_{\mathrm{B}}>\Delta V_{\text {Coul }}$ a junção comporta-se como um resistor.

Esse fenômeno permite algumas aplicações interessantes, como o estabelecimento de um padrão de corrente [22] ou sensíveis amperímetros (eletrômetros) [23]. Como podemos detectar e controlar a entrada ou a saída de um elétron do ponto quântico é possível definir dois estados lógicos, ligado e desligado, respectivamente: esse é o princípio no qual baseiam-se muitos pesquisadores dedicando-se atualmente à construção de um computador em escala atômica (o computador quântico) e de alta velocidade [24].

$\mathrm{Na}$ realidade existe uma outra forma de provocar o tunelamento de elétrons no caso representado na Fig. 3(b) se o potencial químico dos pontos quânticos for aumentado de $\Delta V_{\text {Coul }}$. Para isso, introduzimos um outro terminal ( $\mathrm{B}$, base) e outra bateria $\left(\mathrm{V}_{\mathrm{G}}\right)$ que para melhor visualização estão colocados na Fig. 4(a). Com mais este terminal, completamos o que se pode chamar de um transistor de um único elétron. Imagine agora que $V_{\mathrm{B}}<\Delta V_{\text {Coul }}$ na estrutura, mantendo-se $V_{\mathrm{G}}$ $=0$. Sabemos da discussão anterior que não haverá corrente circulando entre $\mathrm{E}$ e $\mathrm{C}$ e nem entre $\mathrm{C}, \mathrm{E}$ e B. Se $V_{\mathrm{G}} \neq 0$, o que resulta é deslocar as energias do ponto quântico (ou em outras palavras, alterar o potencial químico dos pontos quânticos), causando uma ressonância entre os níveis de $\mathrm{E}$ e $\mathrm{B}$ e os do ponto, permitindo a carga ou a descarga do ponto quântico por um elétron. Se a voltagem $V_{\mathrm{B}}$ é mantida constante e obedecendo à condição $V_{\mathrm{B}}<\Delta V_{\text {Coul }}$, a corrente através dos terminais E-C apresentará máximos representando a passagem dos elétrons, um a um, através do ponto quântico. Na Fig. 4(b) temos a corrente medida no circuito como função de $V_{\mathrm{G}}$ : este comportamento é análogo ao comportamento de um transitor comum [1].

Os primeiros transistores SET foram fabricados por Fulton e Dolan [25] e Kuzmin e Likharev [26] em 1987. As aplicações para um transistor como este são muito amplas, dada a sua operação absolutamente precisa e rápida, como em memórias e portas lógicas. A utilização alternativa de estruturas auto-organizadas para

\footnotetext{
1 Se os pontos quânticos forem substituidos por um poço quântico, ainda que exista o processo de tunelamento, a situação é outra porque a densidade de estados e a dispersão em energia nas direções x e y conseguem acomodar mais elétrons.

${ }^{2}$ Uma possibilidade para isso é um processo de tunelamento de elétrons do terminal E (emissor) para o ponto quântico. O tunelamento é um processo absolutamente quântico, não tendo um análogo clássico (para uma descrição básica sobre tunelamento, ver Ref. [5]).
} 
esse fim, como o sistema InAs/GaAs ou SiGe/Si é bastante promissora devido aos pontos quânticos assim obtidos serem livres de defeitos. Além disso, os dispositivos podem ser desenhados de forma a minimizarem a influência da temperatura na ocupação dos pontos quânticos, como propusemos em um recente trabalho [13].

(a)
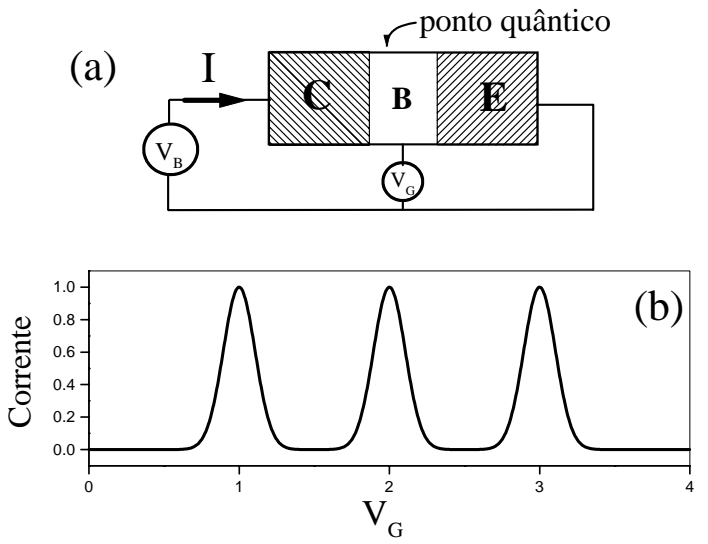

Figura 4. Na parte (a), está uma representação de um transistor SET. A característica da corrente entre coletor e emissor em função da polarização da base é mostrada em (b).

Ao finalizar esta seção, pode surgir uma questão: dada a natureza quântica dos elétrons, como é que fica o Princípio da Incerteza de Heisenberg, quando se diz que foi possível controlar um elétron? Para entender isso, é necessário lembrar que consideramos elétrons que se movem sob um campo elétrico em um material e que a corrente elétrica resultante é determinada pela carga transferida ao material (fio). Aqui não é importante a carga num determinado volume, mas a quantidade de carga que flui pelo fio, ou carga transferida. Esta carga é proporcional à soma dos deslocamentos de todos os elétrons com relação aos átomos da rede cristalina do fio. Como os elétrons podem ser deslocados através do fio por distâncias tão pequenas quanto se queira, a soma acima citada pode ser mudada continuamente e portanto, a carga transferida é contínua. Se uma junção similar àquela da Fig. 4(a) for introduzida no fio, teremos dois comportamentos para os elétrons: um é contínuo, no qual os elétrons se acumularão de um lado da junção, produzindo uma carga total Q; para existir corrente através do circuito, elétrons devem passar através da junção. A Mecânica Quântica mostra que a carga $Q$ somente pode diminuir ou aumentar (dependendo do sentido da corrente) através de um comportamento discreto, quando exatamente um elétron é transferido através do ponto (lembre-se da densidade de estados em um sistema zero-dimensional). Assim, em nenhum momento determinamos a posição e a velocidade do elétron e portanto, o princípio de Heisenberg não foi violado.

\section{Conclusão}

Neste trabalho foram descritas algumas propriedades dos pontos quânticos em conexão com conceitos de quantização e de eletrostática familiares aos cursos de Graduação em Física. Também foi abordado de forma simples a idéia de um transistor baseado nessas estruturas. Como os transistores, que provocaram uma verdadeira revolução há décadas passadas, os pontos quânticos são candidatos bastante promissores para um novo grande passo em ciência e tecnologia.

\section{References}

[1] A. J. Chiquito e F. Lanciotti Jr., Rev. Bras. de Ens. Fís. 20, 309 (1998).

[2] Na verdade, a idéia de uma heteroestrutura semicondutora é bem mais antiga. Já em 1951, W. Schokley propôs a construção de um transistor bipolar mais eficiente com uma junção de materiais semicondutores diferentes; Veja S.M. Sze, Physics of Semiconductor Devices, Wiley, New York (1981).

[3] M. Kastner, Physics Today 46, 24 (1993); M. A. Reed, Scientific American, janeiro 1993; M. Kastner, Ann. Phys. (Leipzig) 9, 885 (2000).

[4] A. J. Chiquito, Rev. Bras. de Ens. Fís. 21, 514 (1999).

[5] R. Eisberg e R. Resnick, Física Quântica, Editora Campus, Rio de Janeiro (1979).

[6] L. J. Challis, Contemporary Physics 33, 111 (1992).

[7] D. F. Holcomb, Am. J. Phys. 67, 278 (1999).

[8] L. Jacak, Eur. J. Phys. 21, 487 (2000).

[9] C. Weissbuch and B. Vinter, Quantum Semiconductor Structures, Academic, New York (1991).

[10] J. Singh, Physics of Semiconductors and their Heterostructures, McGraw-Hill series in Electrical and Computer Engineering, New York (1993).

[11] M. Grundmann, O. Stier and D. Bimberg, Phys. Rev. B 52, 11969 (1995).

[12] J. Tersoff, C. Teichert, and M. G. Lagally, Phys. Rev. Lett. 76, 1675 (1996).

[13] A. J. Chiquito, Yu. A. Pusep, S. Mergulhão, J. C. Galzerani, and N. T. Moshegov, J. Appl. Phys. 88, 1987 (2000).

[14] C. Wasshuber, Ph.D. Thesis, Vienna University of Technology, Áustria (1997).

[15] L. Pauling and E. Wilson, Introduction to Quantum Mechanics, McGraw-Hill, New York (1935).

[16] G. Arfken, Mathematical Methods For Physicists, Academic, New York (1985).

[17] Claude Cohen-Tannoudji, B. Diu and F. Laloë, Quantum Mechanics Vol. 1, Wiley, New York (1977).

[18] N. W. Aschroft and N. D. Mermin, Solid State Physics, Saunders, Fort Worth (1976). 
[19] John R. Reitz, Frederick J. Milford and Robert W. Christy, Fundamentos da Teoria Eletromagnética, Editora Campus, Rio de Janeiro (1982).

[20] R. A. Millikan, Phys. Rev. 32, 349 (1911); H. M. Nussenzveig, Física Básica - Vol.1, Edgard Blücher, São Paulo (1996).

[21] C. J. Gorter, Physica 17, 777 (1951).

[22] J. M. Martinis, M. Nahum, and H. D. Jensen, Phys. Rev. Lett. 72, 904 (1994).
[23] A. N. Korotkov, D. V. Averin, K. K. Likharev, and S. A. Vasenko em H. Koch and H. Lübbig, editores, Single-Electron Tunneling and Mesoscopic Devices, 45, Springer-Verlag, Berlin (1992).

[24] J. R. Tucker, J. Appl. Phys. 72, 4399 (1992).

[25] T. A. Fulton and G. J. Dolan, Phys. Rev. Lett. 59, 109 (1987).

[26] L. S. Kuzmin and K. K. Likharev, JETP Lett. 45, 495 (1987). 